\section{Bright flash on Jupiter in 1983}

SIR - Predictions that Comet Shoemaker-Levy 9 will collide with Jupiter in July 1994 (refs 1, 2) have spurred interest in the observable consequences of the impact ${ }^{3,4}$. The collision is predicted to occur on the far side of Jupiter, thus the most likely instantaneous observable effect will be a flash that momentarily illuminates a satellite located behind Jupiter at that time. We present evidence

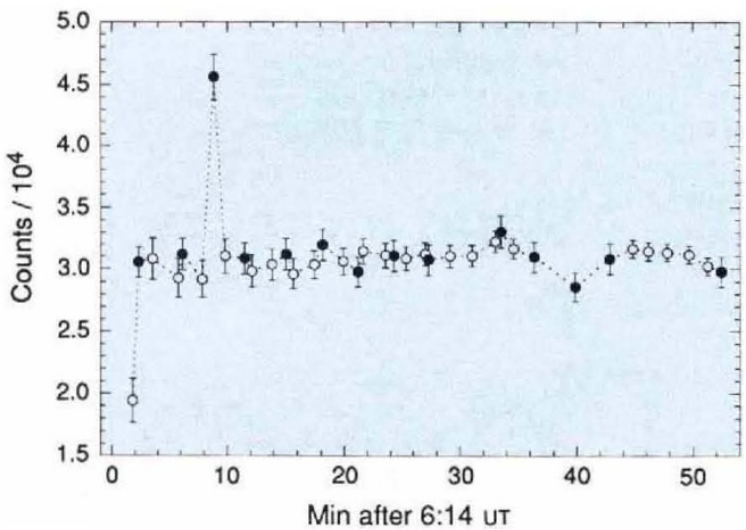

Brightness of Io on 26 July 1983 as it reappeared from Jupiter eclipse. The brightening at 6:22:50 Uт (8.8 min after 6:14 UT) may be evidence for a flash on the far side of Jupiter. Closed circles, data (obtained with the Palomar 1.52-m telescope through a 4,200- $\AA$ filter) from Vidicon images that also included sky background and Europa, which was in front of Jupiter at the time of the observations ${ }^{6}$. The sky and Europa showed no unusual brightening in the frame in which lo was anomalously bright; all pixels containing lo in that frame were 50 per cent higher than the preceding and following frames ${ }^{6}$. Open circles, data (taken with a 0.61-m telescope on Mauna Kea through a 4,400- $\AA$ filter) from disk-integrated photometry ${ }^{5}$, showing no evidence for a flash, constraining the duration of the event to less than $118 \mathrm{~s}$. The data in ref. 6 were normalized to match the data in ref. 5 in the 50 min after 6:14 UT (excluding the anomalous datum in ref. 6 and the first datum in ref. 5 when lo was still in eclipse; eclipse ended at $6: 17 \mathrm{UT})$.

that such a flash on Jupiter's far side may have been detected in 1983 .

On 26 July 1983, we were involved in collecting observations of the jovian moon Io as it reappeared from jovian eclipse $e^{5,6}$. R. M. N. et al. ${ }^{6}$ took a series of $10-\mathrm{s}$ integrations; one of these data showed an anomalous 50 per cent brightening of Io roughly $6 \mathrm{~min}$ after reappearance in one frame (see figure). Several 3-s integrations by H. B. H. et al. ${ }^{5}$ bracketed the anomalous flash, but did not overlap it; they showed no brightening, thus constraining the flash duration to be less than 118 s. Explanations for Io's anomalous brightening include: (1) instrumental effects in the data; (2) an event on Io itself; and (3) illumination of Io by a bright event on the far side of Jupiter.

The data in ref. 6 were Vidicon images containing sky background (scattered light from Jupiter) and the moon Europa - which was in front of Jupiter at the time anomalous brightening.

A flash on the far side of Jupiter could have manifested itself as an increase in Io's brightness. One plausible phenomenon is a 'superbolt' of jovian lightning ${ }^{7}$. Alternatively, calculations of the effects of Shoemaker-Levy 9's impact predict a flash as the comet plunges into the jovian atmosphere. The 50 per cent increase seen here, corresponding to an energy of about $10^{28}$ ergs, would be comparable to a $5-\mathrm{km}$ icy body (with a density of $1 \mathrm{~g} \mathrm{~cm}^{-3}$ ) with a velocity of $60 \mathrm{~km} \mathrm{~s}^{-1}$ converting its kinetic energy to luminous energy in $10 \mathrm{~s}$ with an efficiency of 0.1 . More sophisticated power and energy dissipation estimates for typical cometary bodies predict flashes that would increase a satellite's brightness by 10-30 per cent for a brief interval (less than tens of seconds) $)^{3.4}$. If the flash was caused by an impact, either the body was somewhat larger or faster than a typical comet, or else the luminous efficiency for converting energy to brightness - which is not well known for Jupiter - may be somewhat larger than anticipated. An impact could also have had other observable effects on the jovian atmosphere; we encourage investigators with long-term datasets to check Jupiter during this time period to search for such phenomena.

\section{H. B. Hammel}

\section{Department of Earth, Atmospheric and}

Planetary Sciences,

Massachusetts Institute of Technology,

Cambridge, Massachusetts 02139, USA

R. M. Nelson

Jet Propulsion Laboratory,

California Institute of Technology,

Pasadena, California 91109, USA

1. Nakano, S. IAU Circ. No. 5800 (1993).

2. Chapman, C. R. Nature 363,492-493 (1993)

3. Sekanina, Z. Science 262, 382-387 (1993).

4. Zahnle, K., Mac Low, M.-M. \& Chyba, C. F. Workshop, Lunar and Planetary Laboratory. Tucson (23-24 August 1993).

5. Hammel, H. B. Goguen, J. D. Sinton, W. M. \& Cruikshank, D. P. Icarus 64, 125 (1985)

6. Nelson, R. M. et al. Icarus 101, 223-233 (1993)

7. Borucki, W. J. \& McKay, C. P. Nature 328, 509-510 (1987).

\section{$X$ larger than $Y$}

SIR - The possibility of determining the sex of offspring in humans and animals is a topic of enduring interest. In 1921 it was found that haploid sperm carry either the $\mathrm{X}$ or $\mathrm{Y}$ chromosome ${ }^{1}$, and that after fertilization with the haploid oocyte determine the sex of the offspring. Until now no scientific data have been reported concerning the size differences between $\mathrm{X}$ and $Y$ sperm in humans and animals. We have used polymerase chain reaction (PCR) amplification with primers from the human Y-linked testis-determining gene (SRY; ref. 2) on human blood DNA from 41 male and female subjects. We identified that the primers are suitable for distinguishing between human male and female DNA. We then used the same primers (together with common control primers from the human sperm receptor gene ZP3 on chromosome 7 ; ref. 3) to identify 20 (male and female) single lymphocytes. Our results were $100 \%$ correct.

The same primers were used to determine the sex of 233 non-motile sperm (11 donors), which were individually selected for direct photography before PCR amplification. Two hundred and seventeen sperm $(93.1 \%)$ showed satisfactory amplification, $106(48.8 \%)$ being $\mathrm{Y}$ and $111(51.2 \%) \mathrm{X}$. Under 'blind' conditions, we magnified photographs of individual sperm a further $30 \times$ and directly measured them. Statistical analysis showed the length, perimeter and area of the heads and the length of the neck and tail in $X$ sperm are significantly larger than those of $\mathrm{Y}$ sperm. Thus, in contrast with earlier work ${ }^{4,5}$ our direct methods show for the first 